\title{
Representation Theory and Integration of Nonlinear Spherically Symmetric Equations to Gauge Theories
}

\author{
A. N. Leznov and M. V. Saveliev \\ Institute for High Energy Physics, Serpukhov, USSR
}

\begin{abstract}
A constructive proof of complete integrability of spherically symmetric self-dual equations in Euclidean space $R_{4}$ for an arbitrary embedding of $\mathrm{SU}(2)$ in an arbitrary gauge group $G$ is given on the base of Lax-type representation and representation theory. The equations are solved explicitly for the case of simple Lie groups $G$.
\end{abstract}

1.

The aim of our paper is to give a constructive proof of complete integrability of spherically symmetric classical self-dual equations in $\mathrm{R}_{4}$ for an arbitrary embedding of SU(2) in an arbitrary gauge group $G$ of rank $n$. The technique for dealing with this essentially nonlinear two-dimensional partial differential system involves Lax-type representation [1] (see also [2]) and representation theory, which enable us to reformulate the integrability problem in terms of the main notions of the representation theory of the corresponding group and to solve the equations explicitly. In this the spherically symmetric instantons in $R_{4}$ and monopoles in Minkowski space $R_{3,1}$ (with Higgs Scalar field in adjoint representation of $G$ ) in a classical sense form a special subset of our solutions under the relevant boundary conditions. All our previous results [3] concerning the construction of exact solutions to the spherically symmetric self-dual equations for the minimal embedding of $\mathrm{SU}(2)$ in $G$ with $\prod_{1}^{n} \otimes U(1)$ invariance subgroup are consequences of the general scheme of the present paper.

Note also that in static case the corresponding system of equations for the minimal embedding of SU(2) in $G$ describes generalized (finite, nonperiodic) Toda lattice (see e.g. [4]). In the framework of our consideration [5] the solution to this system as well as its two-dimensional generalization arise as a particular case. 
2.

Let us consider Yang-Mills field configurations $A_{\mu}(x), 0 \leqq \mu \leqq 4$, in $R_{4} \ni x$, spherically symmetric with respect to the total momentum operator $\mathbf{I}=\mathbf{M}+\mathbf{L}$, where $\mathbf{M}=-i \mathbf{x} \times \nabla$ represents space rotations and $\mathbf{L}$ generates some $\mathrm{SU}(2)$ subgroup of $G$. Then there are four operator structures $W^{a}=W^{a}(r, t), o \leqq a \leqq 3, r=\sqrt{\mathbf{x}^{2}}, t=x_{0}$, scalar under the action of total momenta $\mathbf{I},\left[\mathbf{I}, W^{a}\right]_{-}=0$, which parametrize field components $A_{\mu}(x)$,

$$
A_{0}=W^{0}, \quad \mathbf{A}=\mathbf{n} W^{1}+\mathbf{M} W^{2}+\mathbf{n} \times \mathbf{M} W^{3},
$$

where $\mathbf{n}=\mathbf{x} / r$. The electric $\mathbf{E}$ and magnetic $\mathbf{H}$ gauge field strengths are constructed by the ordinary rules, and in the system $\mathbf{n}=(0,0,1)$ have the form

$$
\begin{aligned}
E_{0} & =(2 i)^{-1}\left[D_{z_{+}}, D_{z_{-}}\right]_{-}, \quad H_{0}=(2 i)^{-1}\left\{\left[W^{+}, W^{-}\right]_{-}+h\right\}\left(z_{+}-z_{-}\right)^{-2}, \\
E_{ \pm}-H_{ \pm} & =i\left(z_{+}-z_{-}\right)^{-1}\left(D_{z_{\mp}} W^{ \pm}\right), \quad E_{ \pm}+H_{ \pm}=i\left(z_{+}-z_{-}\right)^{-1}\left(D_{z_{ \pm}} W^{ \pm}\right) .
\end{aligned}
$$

Here

$$
\begin{aligned}
\left\{E_{0}, E_{ \pm}\right\} & \equiv\left\{E_{3}, E_{1} \pm i E_{2}\right\}, \quad\left\{H_{0}, H_{ \pm}\right\} \equiv\left\{H_{3}, H_{1} \pm i H_{2}\right\} ; \\
W_{z_{ \pm}} & \equiv W^{0} \mp i W^{1} ; \\
W^{ \pm} & \equiv-i\left(z_{+}-z_{-}\right)\left\{\left[L^{ \pm}, \pm W^{2}+i W^{3}\right]_{-}-\left(z_{+}-z_{-}\right)^{-1} L^{ \pm}\right\}, \\
L^{ \pm} & \equiv L_{1} \pm i L_{2}, \quad h \equiv i L_{3} ; \quad 2 z_{ \pm} \equiv t \pm i r ; \quad\left(D_{z_{ \pm}} W\right) \equiv W_{z_{ \pm}}-\left[W_{z_{ \pm}}, W\right]_{-}
\end{aligned}
$$

are covariant derivatives. It follows from formulae (2) that $E_{0} \equiv(2 i)^{-1} F_{z_{+} z_{-}}$is gauge field $W_{z_{ \pm}}$tensor in two-dimensional space, $E_{ \pm}$and $H_{ \pm}$are covariant derivatives of the sources $W^{ \pm}$in curved space and $H_{0} \equiv(2 i)^{-1} V$ plays the role of interaction of the sources. The action $S$ and topological charge $Q$ are defined in accordance with (2) by the expressions

$$
\begin{aligned}
& S=\pi \int d z_{+} d z_{-}\left(z_{+}-z_{-}\right)^{2} \operatorname{Sp}\left\{-1 / 2 F_{z_{+} z_{-}}^{2}-1 / 2 V^{2}+\left(z_{+}-z_{-}\right)^{-2}\right. \\
& \left.\cdot\left[\left(D_{z_{+}} W^{-}\right)\left(D_{z_{-}} W^{+}\right)+\left(D_{z_{+}} W^{+}\right)\left(D_{z_{-}} W^{-}\right)\right]\right\}, \\
& Q=-1 / 16 \pi \int d z_{+} d z_{-}\left(z_{+}-z_{-}\right)^{2}
\end{aligned}
$$

$$
\cdot \operatorname{Sp}\left\{F_{z_{+} z_{-}} V-\left(z_{+}-z_{-}\right)^{-2}\left[\left(D_{z_{+}} W^{+}\right)\left(D_{z_{-}} W^{-}\right)-\left(D_{z_{+}} W^{-}\right)\left(D_{z_{-}} W^{+}\right)\right]\right\},
$$

(compare with those of [6] for the SU(2) case). Thus the Lagrangian of the system describes the interaction of charged fields $W^{ \pm}$and gauge field $F_{z_{+} z_{-}}$in curved space, which satisfy the following equations of motion:

$$
\begin{aligned}
{\left[D_{z_{+}}, D_{z_{-}}\right]_{+} W^{ \pm} } & = \pm\left[W^{ \pm}, V\right]_{-}, \\
{\left[D_{z_{+}}, F_{z_{+} z_{-}}\right]_{-} } & =\left[W^{+},\left(D_{z_{+}} W^{-}\right)\right]_{-}-\left[W^{-},\left(D_{z_{+}} W^{+}\right)\right]_{-} .
\end{aligned}
$$

The self-dual equations represent a special subset of general system (4),

$$
\left(D_{z_{\mp}} W^{ \pm}\right)=0, \quad\left[D_{z_{+}}, D_{z_{-}}\right]_{-}=\left[W^{-}, W^{+}\right]_{-},
$$


and arise as a result of equating the components of the electric and magnetic fields $(2)^{1}$. (The anti-self-dual equations can be obtained by the obvious replacement $W^{+} \rightleftarrows W^{-}$.) Owing to the relations $\left[h, D_{z_{ \pm}}\right]_{-}=0,\left[h, W^{ \pm}\right]_{-}= \pm 2 W^{ \pm}$the system (5) reduces to the form

$$
\left[\partial / \partial z_{+}-W_{z_{+}}-W^{+}, \partial / \partial z_{-}-W_{z_{-}}-W^{-}\right]_{-}=0 .
$$

The latter one is the Lax-type representation for the spherically symmetric selfdual Yang-Mills field (compare with [7]) for an arbitrary embedding of SU(2) in $G$, which enables one to fulfil the complete integration of the system.

It is easy to show that the topological charge density $-16 \pi q$ defined by the integrand (3) can be rewritten for the self-dual fịelds in a form

$$
\begin{aligned}
q & =-\operatorname{Sp} h\left[\partial^{2} / \partial z_{+} \partial z_{-}\left(W^{-} W^{+}\right)-\left(W_{z_{+}, z_{-}}-W_{z_{-}, z_{+}}\right)\right] \\
& =-\left[1 / 2 \partial^{2} / \partial z_{+} \partial z_{-}\left(z_{+}-z_{-}\right)^{2}-1\right] \operatorname{Sp}\left[\left(W_{z_{+}, z_{-}}-W_{z_{-}, z_{+}}\right) h\right] .
\end{aligned}
$$

Let us stress that up to now we did not make any assumptions about the properties of the gauge group except that it must contain $S U(2)$ subgroup. This circumstance will allow one to construct in further a wide class of the complete integrable system of two-dimensional nonlinear equations and to obtain its explicit solutions.

\section{3.}

Let us sketch briefly the general scheme of the integration of self-dual system (5) for an arbitrary embedding of SU(2) in a compact simple Lie group $G$ with Lie algebra $\mathfrak{g}$ and Cartan subalgebra $\mathfrak{h}$. The embedding of $\mathrm{SU}(2)$ in $G$ is canonical and determined by its Cartan element $h$ (or embedding vector) [8]. In this the generators $L^{ \pm}$and $h$, which correspond to the subgroup $\mathrm{SU}(2)$, satisfy the usual commutation relations $\left[h, L^{ \pm}\right]_{-}= \pm 2 L^{ \pm},\left[L^{+}, L^{-}\right]_{-}=h$. The subset of the generators corresponding to the positive (negative) roots of $\mathfrak{g}$, which commutes with $h$ will form the invariance subalgebra $\mathfrak{g}_{0}$ together with the elements of $\mathfrak{h}$. The corresponding group will be called invariance subgroup $G_{0} .[$ Note that for the minimal embedding this group is $\prod_{1}^{n} \otimes U(1)$.] Let $Z^{ \pm}\left(\stackrel{\circ}{Z}^{ \pm}\right)$be the maximal nilpotent subgroups in $G\left(G_{0}\right)$ and let $Z_{0}^{ \pm}$be the factor group $Z^{ \pm} / \AA^{ \pm}$. The superscript tilde will appended to the symbol of the corresponding complex hull of these groups, and small letters - for the relevant algebras.

An arbitrary element $g$ of $\tilde{G}$ can be represented by a modified Gaussian decomposition,

$$
g=N_{0}^{+} N_{0}^{-} g_{0}^{N} \equiv M_{0}^{-} M_{0}^{+} g_{0}^{M} .
$$

From $\left(7_{1}\right)$ there follows an identity

$$
\left(M_{0}^{-}\right)^{-1} N_{0}^{+}=M_{0}^{+} g_{0}^{M}\left(g_{0}^{N}\right)^{-1}\left(N_{0}^{-}\right)^{-1},
$$

1 In this it is needed to carry out an additional substitution $W_{z_{+}} \rightarrow W_{z_{+}}-h / 2\left(z_{+}-z_{-}\right)$, $W^{ \pm} \rightarrow-i\left(z_{+}-z_{-}\right) W^{ \pm}$which straightens the space and liquidate the term $-i h / 2\left(z_{+}-z_{-}\right)^{2}$ in $H_{0}$ 
where $\left\{N_{0}^{ \pm}, M_{0}^{ \pm}\right\} \in \tilde{Z}_{0}^{ \pm},\left\{g_{0}^{M}, g_{0}^{N}\right\} \in \tilde{G}_{0}$, owing to which the parameters of elements $M_{0}^{+}, N_{0}^{-}$and $g_{0}^{M}\left(g_{0}^{N}\right)^{-1}$ are the algebraic functions of the group parameters of $M_{0}^{-}$, $N_{0}^{+}$. The representation (6) means the gradientness of the vector $W_{z_{ \pm}}+W^{ \pm}$, i.e.

$$
W_{z_{ \pm}}+W^{ \pm}=g^{-1} \cdot g_{, z_{ \pm}}
$$

The operators $W_{z_{ \pm}}$are spanned by the generators $\left\{z^{ \pm}, h\right\}$, while $W^{ \pm}$are decomposed over the elements corresponding to positive (negative) roots of $\mathfrak{g}$ having the order \pm 2 with respect to $h$, whose totality will be noted by $R_{(2)}^{ \pm}$. In the parametrization $\left(7_{1}\right)$ expression (8) takes the form

$$
\begin{aligned}
W_{z_{+}}+W^{+}= & \left(g_{0}^{M}\right)^{-1}\left(M_{0}^{+}\right)^{-1}\left(M_{0}^{-}\right)^{-1} M_{0, z_{+}}^{-} M_{0}^{+} g_{0}^{M} \\
& +\left(g_{0}^{M}\right)^{-1}\left(M_{0}^{+}\right)^{-1} M_{0, z_{+}}^{+} g_{0}^{M}+\left(g_{0}^{M}\right)^{-1} g_{0, z_{+}}^{M}
\end{aligned}
$$

(there is an analogous relation for $W_{z_{-}}+W^{-}$up to the change $M$ for $N$ ). The absence of generators corresponding to the negative roots of $g / \mathfrak{g}_{0}$ in the decomposition $W_{z_{+}}+W^{+}$spanned by the elements of $\mathfrak{g}$ gives $\left(M_{0}^{-}\right)^{-1} M_{0, z_{+}}^{-}=0$, i.e. the group parameters of $M_{0}^{-}$depend only on $z_{-}, M_{0}^{-} \equiv M_{0}^{-}\left(z_{-}\right)$. By the analogy with it one obtains $N_{0}^{+} \equiv N_{0}^{+}\left(z_{+}\right)$. The requirement that the contributions in (9) relevant to the positive roots of the order unequal to two with respect to $h$ are absence, leads to the system of equations, which connects [due to (7)] the first derivatives of the parameters of $N_{0}^{+}\left(z_{+}\right)$. Thus the realization of these conditions in (9) does not depend on the form of $M_{0}^{-}$, which can be equal to unity, so we have

$$
\left(N_{0}^{+}\right)^{-1} N_{0, z_{+}}^{+}=\sum_{\alpha \in R_{(2)}^{+}} \varphi_{+\alpha}\left(z_{+}\right) X_{\alpha}^{+},
$$

where $X_{\alpha}^{+}$are the elements of $g$ corresponding to the roots $\alpha \in R_{(2)}^{+}$and $\varphi_{+\alpha}\left(z_{+}\right)$are the arbitrary functions. The solution of this system can be presented as $T$-ordered exponent with the Lagrangian $L\left(z_{+}\right) \equiv \sum_{\alpha \in R_{(2)}^{+}} \varphi_{+\alpha}\left(z_{+}\right) X_{\alpha}^{+}$,

$$
\begin{aligned}
& N_{0}^{+}=T \exp \int^{z_{+}} d z_{+} \sum_{\alpha \in R_{(2)}^{+}} \varphi_{+\alpha}\left(z_{+}\right) X_{\alpha}^{+}, \\
& M_{0}^{-}=T \exp \int^{z-} d z_{-} \sum_{\alpha \in R_{(2)}^{+}} \varphi_{-\alpha}\left(z_{-}\right) X_{\alpha}^{-},
\end{aligned}
$$

which is expressed (see, e.g. [9]) in a closed form via repeated integrals of retarded commutators (right-running formula). In this tha final series contains finite number of terms because the group $G$ is finite dimensional. In accordance with $\left(7_{2}\right)$ the group parameters of the elements $M_{0}^{+}, N_{0}^{-}$, and $g_{0}^{M}\left(g_{0}^{N}\right)^{-1}$ can be expressed through the parameters of $N_{0}^{+}, M_{0}^{-}$defined by formulae (11).

In this the expression $\left(3^{\prime}\right)$ for the topological charge density written via the operators $W_{z_{ \pm}}$[entering (8)] reduces after simple algebraic transformations to the form

$$
q=-\left[1 / 2 \partial^{2} / \partial z_{+} \partial z_{-}\left(z_{+}-z_{-}\right)^{2}-1\right] \partial^{2} / \partial z_{+} \partial z_{-}\left[\sum_{\alpha} \operatorname{Sp}\left(h_{\alpha} h\right) \tau_{\alpha}-\operatorname{Sp} h^{2} \ln \left(z_{+}-z_{-}\right)\right]
$$


The parameters $\tau_{\alpha}$ of the Cartan element $\exp \sum_{\alpha} h_{\alpha} \tau_{\alpha}$ of the Gaussian decomposition of $g_{0}^{M}\left(g_{0}^{N}\right)^{-1}$ are expressed through the highest vectors $\xi^{\{1 \alpha\}}$ of the fundamental representation of $G$ with weight

$$
\left\{1_{\alpha}\right\} \equiv\{\underbrace{0, \ldots, 0}_{\alpha-1}, 1,0, \ldots, 0\}, \quad \tau_{\alpha}=\ln \xi^{\left\{1 \alpha_{\alpha}\right\}}\left(\left(N_{0}^{+}\right)^{-1} M_{0}^{-}\right) .
$$

By virtue of this we come to the following final formula for the topological charge density

$$
\begin{aligned}
q= & 1 / 2\left[1 / 2 \partial^{2} \partial z_{+} \partial z_{-}\left(z_{+}-z_{-}\right)^{2}-1\right] \partial^{2} / \partial z_{+} \partial z_{-} \sum_{\alpha, \beta} t_{\beta} \\
& \cdot v_{\alpha} \cdot k_{\alpha \beta} \ln \frac{\xi^{\left\{1_{\alpha}\right\}}\left(\left(N_{0}^{+}\right)^{-1} M_{0}^{-}\right)}{\left(z_{+}-z_{-}\right)^{t_{\alpha}}} .
\end{aligned}
$$

Here $t_{\alpha}$ are the coefficients in the expansion of the Cartan element $h$ over the generators of $\mathfrak{h}, k$ is the Cartan matrix of $\mathfrak{g}$ and $v_{\alpha}$ are the elements of the diagonal matrix $V, V k=k^{T} V$.

Note that analogously with the special case of minimal embedding $\left(G_{0}=\prod_{1}^{n} \otimes U(1)\right)[3]$ our present construction of general solutions to self-dual equations in $R_{4}$ for an arbitrary embedding of SU(2) in $G$ solves also the problem of describing the spherically symmetric monopoles and dyons in $R_{3,1}$ (with Higgs scalar field in adjoint representation) in B.P.S. limit [10]. Namely, the images $\underline{\mathbf{W}}=\mathbf{A}, \underline{W}_{0}= \pm \operatorname{sh} \theta A_{0}$ and $\varphi= \pm \operatorname{ch} \theta A_{0}$ of static sourceless self-dual fields $\left(A_{0}, \mathbf{A}\right)$ in $\dot{R}_{4}$ are dyon (monopole when $\theta=0$ ) solutions in $R_{3,1}$. Thus to construct the nonsingular monopole (dyon) solutions it is needed to take the static limit in (9), $(11)^{2}$ and to provide the finiteness of the energy of the monopole system by the links between the parameters $m_{\alpha}$ and $c_{\alpha}$ in a complete analogy with those of [3].

4.

The construction given in above section solves completely the problem of integration of the spherically symmetric self-dual equations in $R_{4}$ (as well as the equations for the monopole systems in $R_{3,1}$ with Higgs field in adjoint representation in B.P.S. limit) for an arbitrary embedding of SU(2) in gauge group $G$. In this two-dimensional version of generalized (finite, nonperiodic) Toda Lattice described by the system $[3]^{3}$

$$
x_{\alpha, z_{+} z_{-}}=\exp \sum_{\beta=1}^{n} k_{\alpha \beta} x_{\beta}, \quad 1 \leqq \alpha \leqq n, \quad x_{\alpha} \equiv x_{\alpha}\left(z_{+}, z_{-}\right),
$$

2 In this the solutions are characterized by the arbitrary parameters $m_{\alpha}, c_{\alpha}$ but not by the arbitrary functions $\varphi_{ \pm \alpha}\left(z_{ \pm}\right)$

3 The Lagrangian appropriate to system (12) is given by the expression 
arises as a remarkable special case of our general system (5). The solution of (12) for the case when $k$ is the Cartan matrix of simple Lie algebra $g$ can be written in accordance with the general construction in Sect. 3 in a form

$$
\exp \left(-x_{\alpha}\right)=\exp \left\{-\sum_{\beta} \mathrm{k}_{\alpha \beta}^{-1} \ln \left[\varphi_{+\beta}\left(z_{+}\right) \varphi_{-\beta}\left(z_{-}\right)\right]\right\} \cdot \xi^{\left\{1_{\alpha\}}\right\}}\left(\left(N_{0}^{+}\right)^{-1} M_{0}^{-}\right)
$$

which have been previously obtained in [5] in the framework of minimal embedding.

Note that all aforementioned reasonings remain unchanged even in the case of noncompact Lie groups with only exception that one does not succeed in obtaining the solutions of the corresponding system in the notions of the finite dimensional representations ${ }^{4}$. So one has to realize the successive commutation procedure without using the simplifications caused by the existance of the highest vectors. Obviously the presence of this possibility essentially extends the class of the complete integrable equations of the considered type.

A remarkable circumstance is that the system $\tilde{v}_{, z_{+} z_{-}}=\exp \tilde{v}, \omega_{, z_{+} z_{-}}$ $=(\omega+\beta \tilde{v}) \exp \tilde{v}$, which has been integrated recently in [11], is also contained in the above considered class of equations as a special case when $G$ is isomorphic to the group of motions $\mathrm{SO}(3) \bullet T_{3}$. Representation (6) for this group leads to the system $v_{, z_{+} z_{-}}=\exp 2 v, u_{, z_{+} z_{-}}=l(l+1) u \exp 2 v$, where $l$ is an angular momentum value corresponding to the shift operator multiplet in $G=\operatorname{SO}(3) \bullet T_{3}$. The system possesses the following general solution

$$
\begin{aligned}
& u=\left(f_{+}+f_{-}\right)^{l+1}\left[\frac{d^{l}}{d f_{-}^{l}} \frac{\varphi_{-}\left(f_{-}\right)}{\left(f_{+}+f_{-}\right)^{l+1}}+\frac{d^{l}}{d f_{+}^{l}} \frac{\varphi_{+}\left(f_{+}\right)}{\left(f_{+}+f_{-}\right)^{l+1}}\right], \\
& v=1 / 2 \ln \left[f_{+, z_{+}} \cdot f_{-, z_{-}} \cdot\left(f_{+}+f_{-}\right)^{-2}\right],
\end{aligned}
$$

when $l$ is integer. Here $\varphi_{ \pm}\left(f_{ \pm}\right)$and $f_{ \pm}\left(z_{ \pm}\right)$are the arbitrary functions. The solution coincides with those of [11] for $l=1$ if one puts $\tilde{v}=2 v-\ln 1 / 2, \omega=u$ $-\beta(2 v-\ln 1 / 2+1)$. Analogical considerations can be carried out also in more general cases of nonsemisimple groups $G$. In this the relevant systems will contain (5) [or, in particular, (12)] as subsystems corresponding to the simple subgroup of $G$.

The last remark we would like to make is concerned with the generalization of system (12) for the case of an arbitrary numeried matrix $k$. Such a system is encountered in various branches of theoretical physics and mechanics (field theory, solid-state and plasma physics, the theory of electrolytes, aerodynamics, nonlinear optics, cosmological models, etc.). A large number of two-dimensional mathematical physics problems considered by now (Sine-Gordon, Liouville, Korteweg-de Vries, etc.) represents particular issues of this system corresponding to a definite choice of matrix $k$ or its continual generalizations for the case of a continuous spectrum of $\alpha$ index values. It was shown [5] that even in this case it is

4 One should not mix this conclusion with the statement about the equivalence (in a sense) between the integration of the system (12), determined by weight structure of the fundamental representations of the corresponding group, and finite dimensional representation theory of semi-simple Lie groups (see Kostant's paper [4]). Here we have in mind the technical details of calculations, namely, for the noncompact groups. There are no expressions for the solutions via highest vectors in a form like (13) 
possible to construct representation of Lax-type in a form

$$
\begin{aligned}
A_{z_{-}, z_{+}}-A_{z_{+}, z_{-}}+\left[A_{z_{+}, z_{-}}\right]_{-} & =0, \\
A_{z_{ \pm}} & =\sum_{\alpha}\left(u_{\alpha}^{ \pm} h_{\alpha}+f_{\alpha}{ }^{ \pm} X_{\alpha}^{ \pm}\right),
\end{aligned}
$$

where $3 n$ generators $\left\{h_{\alpha}, X_{\alpha}^{ \pm}, 1 \leqq \alpha \leqq n\right\}$ obey the commutation relations

$$
\left[h_{\alpha}, X_{\beta}^{ \pm}\right]_{-}= \pm k_{\beta \alpha} X_{\beta}^{ \pm}, \quad\left[X_{\alpha}^{+}, X_{\beta}^{-}\right]_{-}=\delta_{\alpha \beta} h_{\alpha}, \quad\left[h_{\alpha}, h_{\beta}\right]_{-}=0 .
$$

In this if the functions $u_{\alpha}^{ \pm}= \pm 1 / 2 x_{\alpha, z_{ \pm}}, f_{\alpha}^{ \pm}=\exp 1 / 2(k x)_{\alpha}$, then (14) follows from $(15)^{5}$, while without those constraints on $u_{\alpha}^{ \pm}, f_{\alpha}^{ \pm}$representation (14) gives

$$
\left(\ln f_{\alpha}^{ \pm}\right)_{, z_{\mp}}=\mp\left(k u^{\mp}\right)_{\alpha}, \quad u_{\alpha, z_{-}}^{+}-u_{\alpha, z_{+}}^{-}=f_{\alpha}^{+} f_{\alpha}^{-},
$$

from which, introducing the functions $\varrho_{\alpha} \equiv \ln f_{\alpha}^{+} f_{\alpha}^{-}$, one obtains

$$
\varrho_{\alpha, z_{+} z_{-}}=\sum_{\beta} k_{\alpha \beta} \exp \varrho_{\beta} .
$$

The functions $\varrho_{\alpha}$ are linearly independent for nondegenerate matrices $k$ [in this $\left.x_{\alpha}=\left(k^{-1} \varrho\right)_{\alpha}\right]$, while in the opposite case they satisfy the relations $\left(\varrho \cdot \kappa^{i}\right)=0$, where $\kappa_{\alpha}^{i}$ are the LHS eigenvectors of matrix $k$ with zero eigenvalues. Thus in the scheme (14-16) under consideration together with the Cartan matrices of simple Lie algebras the generalized Cartan matrices of infinite-dimensional contragredient Lie algebras, whose properties have been described in [12] can also be committed. In particular, for the generalized Cartan matrices of the 2-nd order in these algebras, namely, $\left(\begin{array}{rr}2 & -2 \\ -2 & 2\end{array}\right)$ and $\left(\begin{array}{rr}2 & -1 \\ -4 & 2\end{array}\right)$ system (16) automatically reduce to the equations $\varrho_{, z_{+} z_{-}}=2 \exp \varrho-2 \exp (-\varrho)$ and $\varrho_{, z_{+} z_{-}}=2 \exp \varrho-\exp (-2 \varrho)$, respectively ${ }^{6}$. These equations possess very remarkable symmetric properties [13], which are characteristic just for infinite-dimensional algebras.

We wish to thank B. A. Arbuzov, O. A. Khrustalev, A. A. Kirilov, Yu. I. Manin, M. A. Mestvirishvili, S. P. Novikov, and A. B. Shabat for the useful discussions.

5 The existence of $n$ independent integrals of motions, which are in involution, as it was shown by Flaschka [4] for $A_{n}$, for system (12) in one-dimensional case is the evident consequence of (14-15). Really, it is easy to check that in static limit the successive powers of the operator $L(r)$ $=A_{z_{+}}-A_{z_{-} I_{+_{+}}+z_{-}}$are the integrals. These eigenvalues are expressed only via parameters $m_{\alpha}, 1 \leqq \alpha \leqq n$, characterizing solution (13) [in this $\left.\varphi_{+\alpha}\left(z_{+}\right)=c_{\alpha} \exp m_{\alpha} z_{+}, \varphi_{-\alpha}\left(z_{-}\right)=c_{\alpha}^{*} \exp ^{-} m_{\alpha} z_{-}\right]$. To calculate $\mathrm{Sp} L^{p}(t)$ it is quite enough to consider the asymptotic behaviour of the function (13) at $r \rightarrow \infty$ as well as in calculation of the monopole magnetic mass matrix. Thus the problem is in fact analogous to the calculation of Casimir operators eigenvalues for the corresponding simple Lie groups

6 The system describing Toda lattice with periodic boundary conditions is also related with these algebras

\section{References}

1. Lax, P.L.: Comm. Pure Appl. Math. 21, 467-491 (1968)

2. Faddeev, L.D. : Sovrem. Problemy Mat. 3, 93 (1974); Manin, Yu.I. : Sovrem. Problemy Mat. 11, 1 (1978); Dubrovin, B.A., Matveev, V.B., Novikov, S.P.: Usp. Mat. Nauk 31, 55-136 (1976); Zakharov, V.E.: Lecture notes in mathematics. Berlin, Heidelberg, New York: Springer 1978 
3. Leznov, A.N., Saveliev, M.V.: Phys. Lett. 79B, 294-296 (1978); 83B, 314-316 (1979); Lett. Math. Phys. 3, 207-211 (1979); Preprint IHEP 78-177, Serpukhov (1978); Leznov, A.N.: Preprint IHEP 79-12, Serpukhov (1979)

4. Toda, M.: Phys. Reps. 18C, 1 (1975); Henon, M.: Phys. Rev. B 9, 1921-1923 (1974); Flaschka, H.: Phys. Rev. B 9, 1924-1925 (1974); Manakov, S.V.: Zh. Eksp. Teor. Fiz. 67, 543-555 (1974); Bogoyavlensky, O.I.: Commun. Math. Phys. 51, 201-207 (1976); Hermann, R.: Interdisciplinary Math., Math. Soc. Press. 15 A, 18 B; Olshanetzki, M.A., Perelomov, A.M.: Preprint ITEP 139 (1978); Moser, J.: Lecture notes in mathematics, Vol. 38, 467 (1975); Kostant, B.: Preprint MIT (1979)

5. Leznov, A.N., Saveliev, M.V.: Lett. Math. Phys. 3, 489-494 (1979)

6. Witten, E.: Phys. Rev. Lett. 38, 121 (1977)

7. Belavin, A.A., Zakharov, V.E.: Pisma v Zh. Eksp. Teor. Fiz. : 25, 603-607 (1977)

8. Gantmakher, F.R.: Mat. Sb. 5, 101 (1939); Dynkin, E.B. : Mat. Sb. 30, 349 (1952); Kostant, B. : Am. J. Math. 81, 973 (1959)

9. Bogolubov, N.N., Shirkov, D.V.: Introduction to the theory of quantized fields. New York: Interscience 1959

10. Bogomolny, E.B.: Sov. J. Nucl. Phys. 24, 449 (1976); Prasad, M.K., Sommerfield, C.M.: Phys. Rev. Lett. 35, 760 (1975)

11. Ibragimov, N. Kh., Jiber, A.V., Shabat, A.B.: Preprint (1979)

12. Kac, V.G.: Izv. Akad. Nauk SSSR, Ser. Matem. 32, 1323 (1968); 34, 385 (1970); Moody, R.V.: Bull. Am. Math. Soc. 13, 217 (1967)

13. Ibragimov, N. Kh., Shabat, A.B.: Funkcional Anal. i Prilozen 13, 27 (1979); Jiber, A.V., Shabat, A.B. : Dokl. Akad. Nauk SSSR 247, 1103-1105 (1979)

Communicated by Ya. G. Sinai

Received August 5, 1979 